CARDIOVASCULAR MEDICINE

\title{
Novel quantitative assessment of myocardial perfusion by harmonic power Doppler imaging during myocardial contrast echocardiography
}

\author{
S Yamada, K Komuro, T Mikami, N Kudo, H Onozuka, K Goto, S Fujii, K Yamamoto, A Kitabatake
}

Heart 2005;91:183-188. doi: 10.1136/hrt.2004.035857

See end of article for authors' affiliations

Correspondence to: Dr Satoshi Yamada, Department of Cardiovascular Medicine, Hokkaido University Graduate School of Medicine, Kita-15, Nishi-7, Kita-ku, Sapporo 060-8638, Japan; syamada@med.hokudai. ac.jp

Accepted 24 May 2004

\begin{abstract}
Objective: To test the hypothesis that the power of the received signal of harmonic power Doppler imaging (HPDI) is proportional to the bubble concentration under conditions of constant applied acoustic pressure, and to determine whether a new quantitative method can overcome the acoustic field inhomogeneity during myocardial contrast echocardiography (MCE) and identify perfusion abnormalities caused by myocardial infarction.

Methods: The relation between Levovist concentration and contrast signal intensity (Cl) of HPDI was investigated in vitro under conditions of constant acoustic pressure. MCE was performed during continuous infusion of Levovist with intermittent HPDI every sixth cardiac cycle in 11 healthy subjects and 25 patients with previous myocardial infarction. In the apical views myocardial $\mathrm{Cl}\left(\mathrm{Cl}_{\text {myo }}\right)$ was quantified in five myocardial segments. The $\mathrm{Cl}$ from the left ventricular blood pool adjacent to the segment was also measured in $\mathrm{dB}$ and subtracted from the $\mathrm{Cl}_{\text {myo }}$ (relative $\mathrm{Cl}(\operatorname{Re} \mid \mathrm{Cl})$ ).

Results: $\mathrm{Cl}$ had a logarithmic correlation and the calculated signal power a strong linear correlation with Levovist concentration in vitro. Thus, a difference in $\mathrm{Cl}$ of $\mathrm{X} \mathrm{dB}$ indicates a microbubble concentration ratio of $10^{\mathrm{x} / 10}$. In normal control subjects, $\mathrm{Cl}_{\text {myo }}$ differed between the five segments $(\mathrm{p}<0.0001)$, with a lower $\mathrm{Cl}_{m y o}$ in deeper segments. However, RelCl did not differ significantly between segments $(p=0.083)$. RelCl was lower $(p<0.0001)$ in the 39 infarct segments (mean (SD) - $18.6(2.8) \mathrm{dB})$ than in the 55 normal segments (mean (SD) - 15.1 (1.6) dB). RelCl differed more than $\mathrm{Cl}_{\text {myo }}$ between groups.

Conclusions: The new quantitative method described can overcome the acoustic field inhomogeneity in evaluation of myocardial perfusion during MCE. RelCl represents the ratio of myocardium to blood microbubble concentrations and may correctly reflect myocardial blood volume fraction.
\end{abstract}

M icrobubble concentration within tissue during myocardial contrast echocardiography (MCE) reflects the myocardial blood volume per unit volume (that is, myocardial blood volume fraction). ${ }^{12}$ The contrast signal intensity (CI) on greyscale B mode harmonic imaging after intravenous injection of ultrasound contrast agents has been suggested to reflect the microbubble concentration ${ }^{3}$ and is used clinically as a quantitative measure to allow estimation of myocardial blood volume. ${ }^{2-8}$ Harmonic power Doppler imaging (HPDI), which has advantages over B mode imaging in opacification of the myocardium, has been used during MCE. ${ }^{9-14}$ Although animal experiments have shown that the severity of coronary stenosis can be assessed by quantitative analysis of HPDI, ${ }^{15}$ some problems remain regarding quantitative assessment of myocardial blood volume with HPDI: the lack of detailed information regarding the relation between microbubble concentration and CI of HPDI; and the inhomogeneity of the insonified ultrasound field during MCE. As CI is dependent on the acoustic pressure, ${ }^{16-18}$ comparison of myocardial CI $\left(\mathrm{CI}_{\mathrm{myo}}\right)$ between myocardial regions, to which different pressures must be applied, is of only limited value.

We hypothesised that the power of the received signal of HPDI is proportional to the bubble concentration under conditions of constant applied acoustic pressure. During MCE, applied acoustic pressure may be similar between a given myocardial region and the adjacent intracavity blood pool. Therefore, $\mathrm{CI}_{\text {myo }}$ can be calibrated by using the CI from the adjacent blood pool to quantify the ratio (myocardium to blood) of microbubble concentrations. This study was performed to test our hypothesis in vitro and to determine whether our new quantitative method can overcome the acoustic field inhomogeneity during MCE and identify perfusion abnormalities caused by myocardial infarction.

\section{METHODS}

\section{Microbubble concentration and $\mathrm{Cl}$ of HPDI in vitro}

Microbubble solutions of the ultrasound contrast agent Levovist, consisting of galactose based, air filled microbubbles (Schering AG, Berlin, Germany), were prepared at concentrations of $1,2,8,32$, and $128 \mathrm{mg} / \mathrm{l}$ by mixing with 1 litre of distilled water in glass beakers. The bubbles thus produced were mixed constantly by a magnetic stirrer. A transducer was fixed to the upper surface of a cylindrical jerry block $5 \mathrm{~cm}$ in height fixed in the centre of the beaker. Harmonic Doppler images were recorded by a Sonos 5500 (Philips Medical Systems, Andover, Massachusetts, USA) with a broadband harmonic transducer ( $\mathrm{S} 3$ probe), with transmission at $1.3 \mathrm{MHz}$ and reception at $2.6 \mathrm{MHz}$. The displayed dynamic range was $40 \mathrm{~dB}$. The image angle was set at $15^{\circ}$, with a focal point of $6 \mathrm{~cm}$, pulse repetition frequency of $4.6 \mathrm{kHz}$ (maximum), and a medium packet size. The microbubble solutions were exposed to different transmission powers with mechanical indices (MIs) of 0.6 and 1.0. On the basis of the results of preliminary experiments, Doppler gain was adjusted to $20 \%$ for MI of 0.6 and $0 \%$ for MI of 1.0 to

Abbreviations: $\mathrm{AU}$, acoustic unit; $\mathrm{Cl}$, contrast signal intensity; $\mathrm{Cl}_{\text {blood, }}$ contrast intensity in the region of interest placed at the left ventricular cavity adjacent to the myocardial region of interest; $\mathrm{Cl}_{\text {myo }}$, myocardial contrast intensity; HPDI, harmonic power Doppler imaging; MCE, myocardial contrast echocardiography; $\mathrm{Ml}$, mechanical index; RelCl, relative contrast intensity; $\mathrm{ROI}$, region of interest; SPECT, single photon emission computed tomography 
avoid both signal saturation and lack of opacification. To minimise the destruction of microbubbles in the 1 litre solution, the solution was scanned with each MI only once. For each microbubble concentration, five datasets were obtained from different solutions. Data were recorded on 5 inch magneto-optical disks.

Images were analysed digitally by a QuantiCon system (GE Medical Systems, Milwaukee, Wisconsin, USA). The mean CI of HPDI in the user defined region of interest (ROI) was measured in $\mathrm{dB}$, acoustic units (AUs), or as the square of AU $\left(\mathrm{AU}^{2}\right)$. $\mathrm{AU}$ is derived from the value in $\mathrm{dB}(\mathrm{X})$ as $255 \times 10^{(\mathrm{X}-\mathrm{DR}) / 20}$, where $\mathrm{DR}$ is the dynamic range. AU ranges from $0-255$ and is theoretically proportional to the amplitude of the received signal. Therefore, $\mathrm{AU}^{2}$ is theoretically proportional to the power of the harmonic power Doppler signal. At the deep parts of the microbubble solution, acoustic pressure may be decreased because of attenuation of ultrasound by the presence of microbubbles in the path of the ultrasound beam. Therefore, CI was measured in a rectangular ROI $2.5 \mathrm{~mm}$ in height placed just below the border between the jerry and the solution, where the applied pressure was considered constant (fig l).

\section{Subjects of a clinical study}

Eleven healthy subjects (mean (SD) 35 (9) years old, 10 men) and 25 consecutive patients with previous myocardial infarction (mean (SD) 65 (10) years old, 20 men) were enrolled in the present study. Myocardial infarction was confirmed by the presence of a Q wave in two or more ECG leads. Inclusion criteria of the patients were the presence of severe asynergy in the apical two or four chamber view on resting echocardiography, and the presence of defective or reduced perfusion on resting single photon emission computed tomography (SPECT) in the segments corresponding to those with echocardiographic wall motion abnormalities. Subjects with acute myocardial infarction within three months, unstable angina, pregnancy, or lactation were excluded from the present study. Coronary angiography was not a prerequisite for this study. The study protocol was approved by our institutional ethics committee. Informed consent was obtained from all subjects before participation in the study.

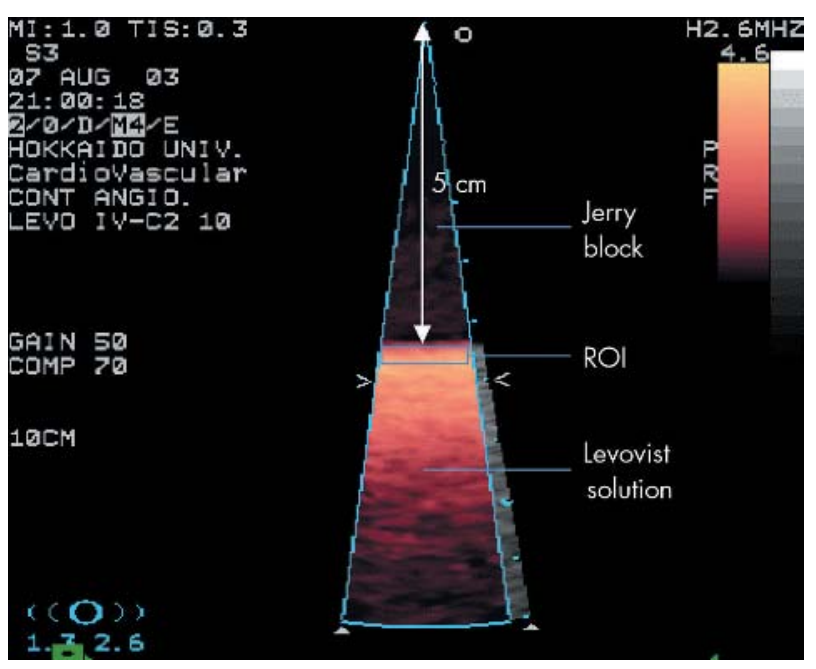

Figure 1 Harmonic power Doppler image of Levovist solution. The contrast signal intensity (CI) decreased in proportion to depth of the solution due to ultrasound attenuation by the presence of microbubbles. The region of interest (ROI) was placed just below the border between the jerry block and the solution to measure $\mathrm{Cl}$ under conditions of constant applied acoustic pressure.

\section{Myocardial contrast echocardiography}

MCE was performed in normal subjects to determine whether the new quantitative method could overcome the inhomogeneous opacification between myocardial regions. In patients with myocardial infarction, MCE was performed within two weeks of SPECT.

HPDI (transmit 1.3, reception $2.6 \mathrm{MHz}$ ) was performed with the same ultrasound system. The MI was set at 1.6, with a pulse repetition frequency of $3.7-4.2 \mathrm{kHz}$ (maximum) and a medium packet size. The focus was placed at the mid level of the left ventricular cavity. Before contrast administration, the upper limit of Doppler gain was determined to avoid motion induced artefacts. The apical four chamber view was used in normal subjects and the apical two or four chamber view was used in each patient with myocardial infarction based on the location of the wall motion abnormality determined on resting echocardiography. Levovist $(300 \mathrm{mg} /$ $\mathrm{ml}$ ) was administered into the right antecubital vein by continuous infusion at a rate of $2.5 \mathrm{ml} / \mathrm{min}$. Two minutes after commencement of the infusion, images of intermittent HPDI were acquired by ultrasound transmission gated to end systole every sixth cardiac cycle. For accurate measurement of the signals from both the myocardium and the blood pool, two types of Doppler gain were used. The higher gain was optimised visually to obtain sufficient opacification of the intracavity blood adjacent to the basal segments and the lower gain was optimised to avoid saturation of signals from the blood pool adjacent to the apical myocardium. Images were recorded on magneto-optical disks.

\section{Analysis of HPDI}

Images were analysed by a Quanticon system. The left ventricular wall in the apical two and four chamber views was divided into five segments: basal inferior/septal, midinferior/septal, apical, mid-anterior/lateral, and basal anterior/lateral. An ellipsoidal ROI was placed over every myocardial segment on each image and mean $\mathrm{CI}_{\text {myo }}$ was measured in $\mathrm{dB}$. Then the $\mathrm{CI}$ in the ROI placed at the left ventricular cavity adjacent to the myocardial ROI $\left(\mathrm{CI}_{\text {blood }}\right)$ was also measured in $\mathrm{dB}$ and subtracted from the $\mathrm{CI}_{\text {myo }}$. This value, relative CI (RelCI), represents the ratio of microbubble concentrations between the myocardium and the adjacent blood pool, if our hypothesis regarding the relation between the bubble concentration and CI is correct. As signals from the blood pool are much stronger than those from the myocardium, saturation of the blood pool should be avoided for accurate measurement of RelCI. Insufficient opacification of the myocardium, which is often seen in the far imaging field, should also be avoided. To eliminate such inappropriate conditions, we decided that $\mathrm{CI}_{\text {blood }}$ of 20 to $36 \mathrm{~dB}$ was acceptable. If the $\mathrm{CI}_{\text {blood }}$ was beyond the limits on every image, the segment was excluded from further analysis.

\section{SPECT}

In each patient with myocardial infarction, $600 \mathrm{MBq}$ of technetium-99m sestamibi was injected at rest and images were recorded after one hour. SPECT images were acquired by a rotating dual detector gamma camera with the detectors mounted at right angles and fitted with high resolution collimators (VXHR, Vertex; ADAC Laboratories, Milpitas, California, USA). Sixty four projections of 40 seconds were acquired over a $180^{\circ}$ orbit. Data were processed with ramp and low pass filters. After back projection, tomographic images were reconstructed in the horizontal and vertical long axis views. Counts within a myocardial segment in each image were normalised to the highest counts within that image and colour coded. SPECT images were interpreted by two observers blinded to all other information. The left ventricular wall was divided into five segments corresponding to 

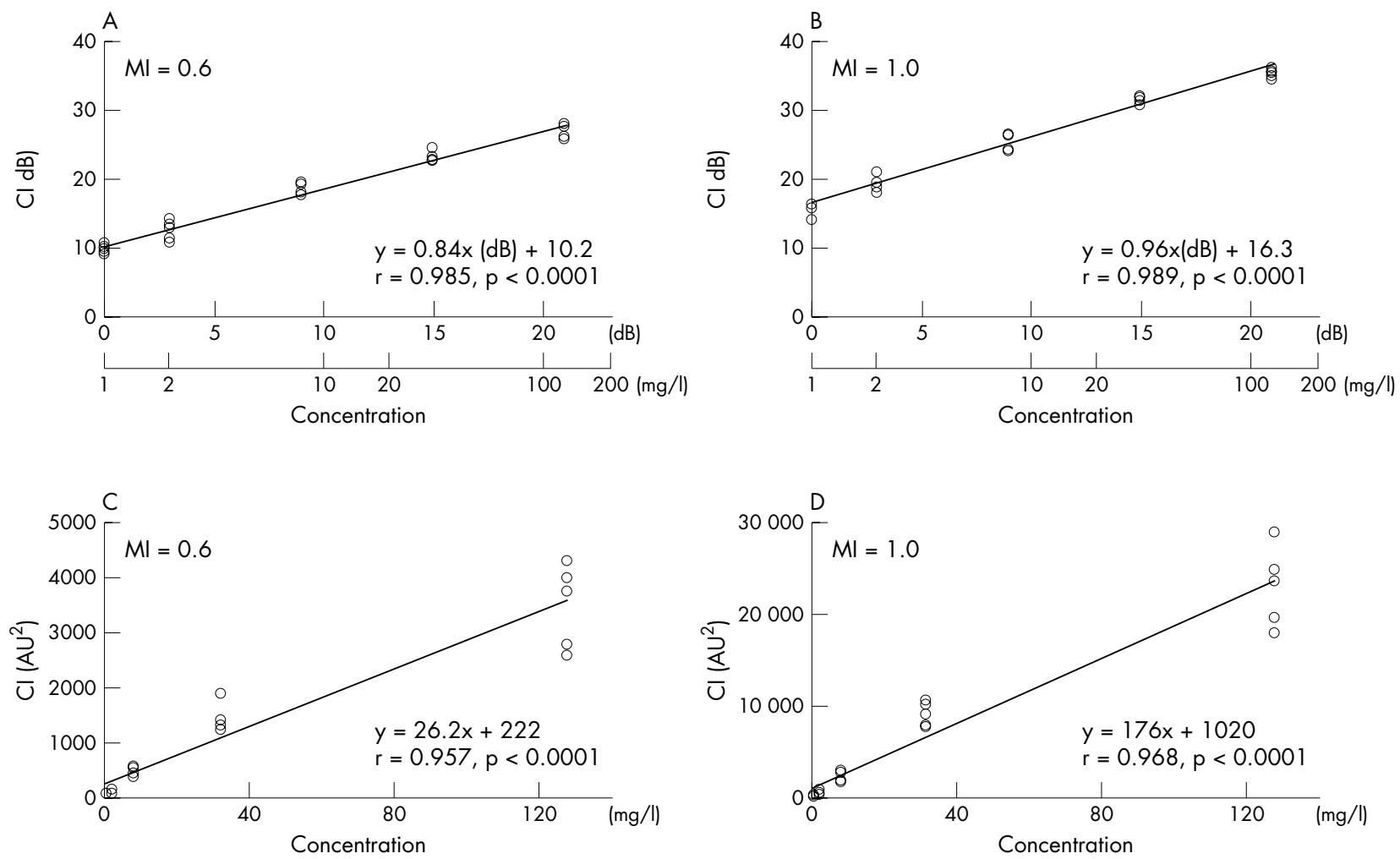

Figure 2 Relations between Levovist concentration and $\mathrm{Cl}$ in $\mathrm{dB}$ with mechanical index (MI) of (A) 0.6 and (B) 1.0, and between the concentration and $\mathrm{Cl}$ in squared acoustic units $\left(A U^{2}\right)$ with $\mathrm{MI}$ of (C) 0.6 and (D) 1.0. In $\mathrm{A}$ and $\mathrm{B}$, the concentration is expressed in logarithmic scale where the value in $\mathrm{dB}$ was calculated as $10 \times \log ($ concentration). In addition, $\mathrm{Cl}$ in $\mathrm{dB}$ can be regarded as the log converted value of the power of the ultrasound signal as $10 \times \log ($ power)

those in echocardiographic analysis. Myocardial perfusion was classified as normal, reduced, and defective.

\section{Statistical analysis}

Data are expressed as mean (SD). One way analysis of variance was used to estimate the significance of differences in the indices between the five segments in the normal subjects. Differences between the two segments were assessed by the paired $t$ test. Variables were compared between the normal myocardial segments and the infarct segments by the unpaired $t$ test. Differences were considered significant at $\mathrm{p}<0.05$.

\section{RESULTS}

Relation between microbubble concentration and $\mathrm{Cl}$

$\mathrm{CI}$ in $\mathrm{dB}$ increased linearly with microbubble concentration in a logarithmic scale (fig 2A, B). The power of the received signal is theoretically proportional to the bubble concentration, and thus the theoretical slope of the CI in $\mathrm{dB}$ plotted against the concentration in a logarithmic scale as $10 \times$ $\log$ (concentration) should be 1.0. Best fitted slopes for MIs of 0.6 and 1.0 were 0.84 and 0.96 , respectively. There was an excellent linear correlation between bubble concentration and $\mathrm{CI}$ in $\mathrm{AU}^{2}$ (fig $2 \mathrm{C}, \mathrm{D}$ ), indicating that the theoretical relation between bubble concentration and CI is valid for
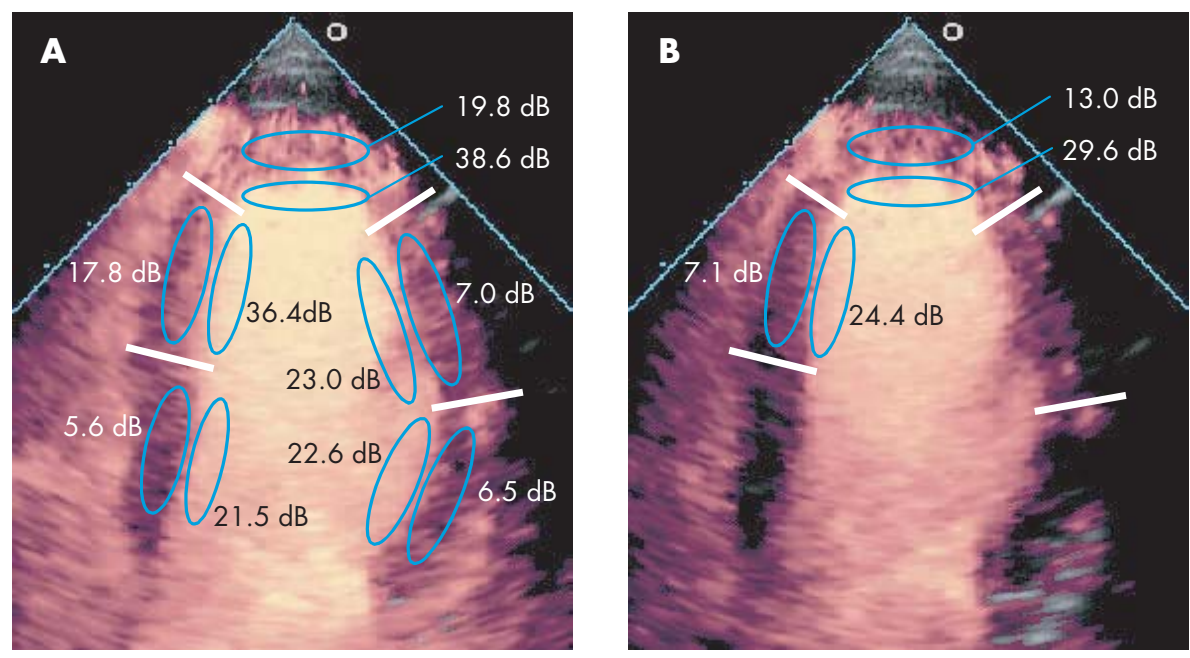

Figure 3 Representative results of measurement of relative $\mathrm{Cl}(\mathrm{Re} \mathrm{Cl})$. Images obtained (A) at the higher Doppler gain of $65 \%$ and $(B)$ at the lower Doppler gain of $45 \%$ were analysed. The RelCls were calculated as $-15.9 \mathrm{~dB}$ in the basal septal segment, $-17.3 \mathrm{~dB}$ in the mid-septal segment, $-16.6 \mathrm{~dB}$ in the apical segment, $-16.0 \mathrm{~dB}$ in the mid-lateral segment, and $-16.1 \mathrm{~dB}$ in the basal lateral segment. 

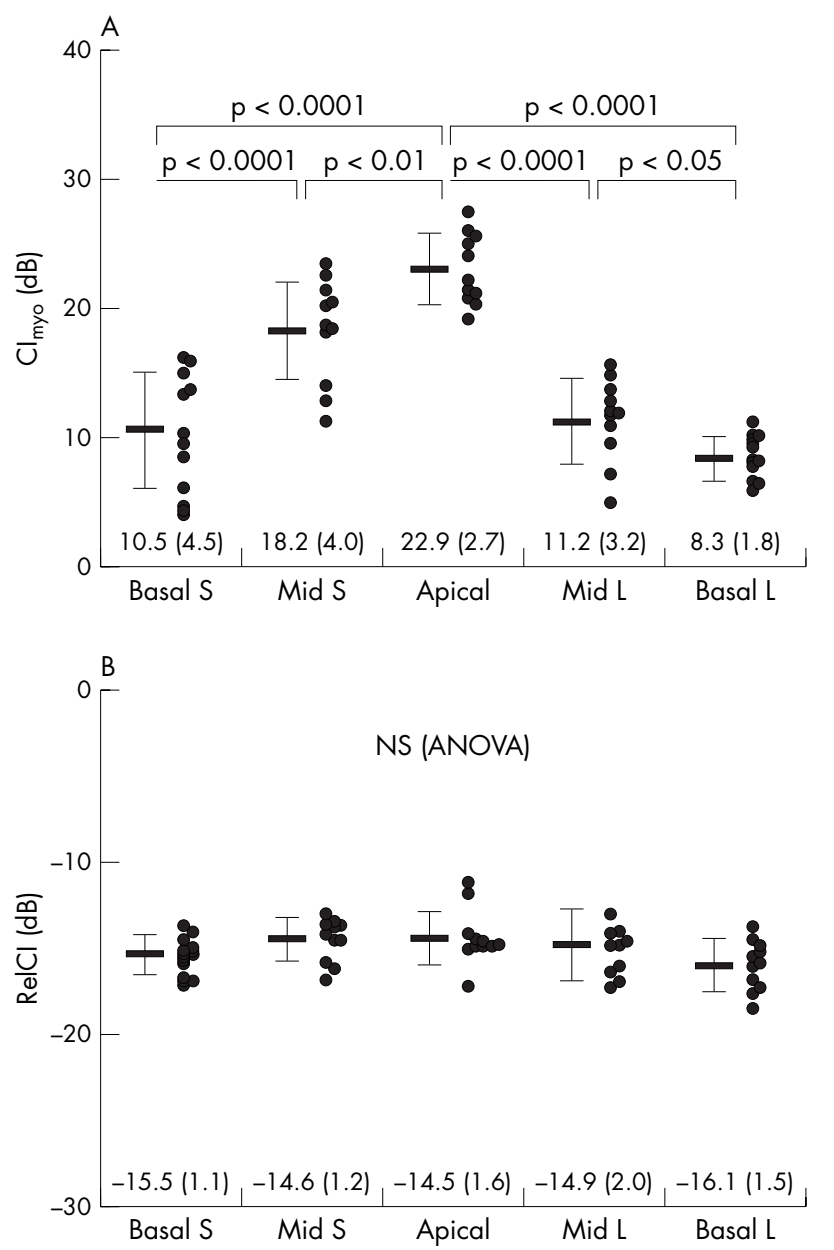

Figure 4 (A) Myocardial $\mathrm{Cl}\left(\mathrm{Cl}_{\text {myo }}\right)$ and (B) $\mathrm{RelCl}$ in normal control subjects. Apical, apical segment; Basal L, basal lateral segment; Basal S, basal septal segment; Mid L, mid-lateral segment; Mid S, mid-septal segment. ANOVA, analysis of variance; NS, not significant.

HPDI in conjunction with Levovist. Thus, a difference in CI of $\mathrm{X} \mathrm{dB}$ under conditions of constant acoustic pressure indicates a ratio of microbubble concentration of $10^{\mathrm{X} / 10}$-for example, an increase in $\mathrm{CI}$ of $3 \mathrm{~dB}$ indicates doubling of the ratio of bubble concentration.

\section{Clinical study}

Figure 3 shows representative contrast images obtained in a normal control subject. Firstly, $\mathrm{CI}_{\text {myo }}$ and $\mathrm{CI}_{\text {blood }}$ were measured in each segment on the image at higher Doppler gain. In the mid-septal and apical segments, $\mathrm{CI}_{\text {blood }}$ was more than $36 \mathrm{~dB}$. Accordingly, these two segments were measured again on the image at lower Doppler.

In all of the 55 segments in the 11 normal subjects $\mathrm{CI}_{\text {blood }}$ values at either higher or lower Doppler gain were within the defined limits of 20 to $36 \mathrm{~dB}$ and no data were excluded from the analysis. The $\mathrm{CI}_{\text {myo }}$ values measured on images at higher Doppler gain differed between the five segments ( $\mathrm{p}<0.0001$, analysis of variance $): \mathrm{CI}_{\text {myo }}$ was lower in the segments located deeper in the ultrasound field (fig 4). However, RelCI did not differ between the five segments $(\mathrm{p}=0.083$, analysis of variance) (fig 4 ) and varied less than $\mathrm{CI}_{\text {myo }}$ in each segment.

In the 25 patients with previous myocardial infarction, 43 segments were akinetic or severely hypokinetic on resting echocardiography. Twelve of these were basal inferior/septal, nine mid-inferior/septal, 16 apical, five mid anterior/lateral segments, and one basal anterior segment. In four of these 43 segments (9\%; one basal inferior, one basal septal, and two apical) $\mathrm{CI}_{\text {blood }}$ was lower than $20 \mathrm{~dB}$ even on the image taken at higher Doppler gain, and these segments were excluded from the analysis. $\mathrm{CI}_{\text {myo }}$ and RelCI were measured in the remaining 39 infarct segments in 24 patients and the values were compared with those in the 55 normal segments in the normal control subjects (fig 5). $\mathrm{CI}_{\text {myo }}$ was significantly lower in the infarct segments than in the normal segments but there was considerable overlap between the groups. RelCI differed more between the infarct and normal segments than $\mathrm{CI}_{\text {myo }}$.

\section{DISCUSSION}

The relation between microbubble concentration and CI of HPDI was investigated under conditions of constant applied acoustic pressure. The results indicated that the values of CI measured in $\mathrm{dB}$ and in $\mathrm{AU}^{2}$ (CI measured in $\mathrm{AU}^{2}$ represents received signal power) are correlated logarithmically and linearly with Levovist concentration, respectively. We used this validated relation to calibrate $\mathrm{CI}_{\text {myo }}$ during $\mathrm{MCE}$ by $\mathrm{CI}_{\text {blood }}$ from the adjacent blood pool to estimate the ratio of
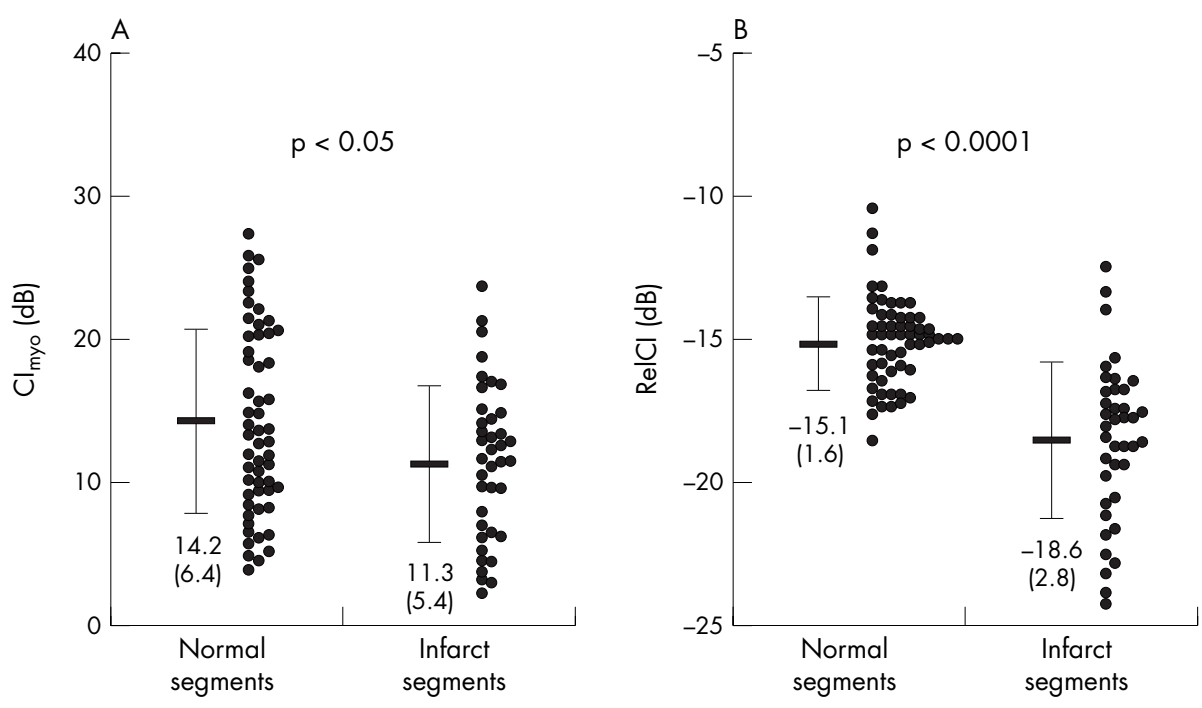

Figure 5 (A) $\mathrm{Cl}_{\text {myo }}$ and (B) $\mathrm{RelCl}$ in normal and infarct segments. 
myocardium to blood microbubble concentrations. In normal control subjects, myocardial opacification varied between regions because of acoustic field inhomogeneity, whereas the calibrated index RelCI varied much less both between myocardial segments and between individual subjects. We also observed reduced RelCI in the infarcted myocardium in patients with chronic coronary artery disease. These observations indicated that this new method can overcome the acoustic field inhomogeneity in quantitative evaluation of myocardial perfusion during MCE.

\section{Microbubble concentration and $\mathrm{Cl}$ of HPDI}

Theoretically, relative changes in microbubble concentration produce corresponding changes of equal magnitude in the scattered intensity. ${ }^{19}$ Schwarz and colleagues ${ }^{19}$ showed previously that pulsed wave Doppler audio intensity (the square of the voltage) is proportional to the relative concentration of Levovist. Although they observed relative changes in microbubble concentration of only 4.6-fold, we observed changes in CI of HPDI corresponding to the relative changes in Levovist concentration of up to 128 -fold. Consistent with the results of pulsed wave Doppler experiments reported by Schwarz and colleagues, our findings indicated that the theoretical relation between bubble concentration and CI is also valid for HPDI in conjunction with Levovist.

CI measured in $\mathrm{dB}$, the log compressed value of the power of the received signal as $10 \times \log$ (power), correlated logarithmically with microbubble concentration. Therefore, the difference in $\mathrm{CI}$ in $\mathrm{dB}$ should be regarded as the ratio of microbubble concentration as follows: $\mathrm{C}_{1} / \mathrm{C}_{2}=$ $10^{\mathrm{X}_{1} / 10} / 10^{\mathrm{X}_{2} / 10}=10^{\left(\mathrm{X}_{1}-\mathrm{X}_{2}\right) / 10}$, where $\mathrm{C}_{1}$ and $\mathrm{C}_{2}$ are the microbubble concentrations at sites $\mathrm{l}$ and 2 , respectively, and $\mathrm{X}_{1}$ and $\mathrm{X}_{2}$ are $\mathrm{CI}$ in $\mathrm{dB}$ at sites $\mathrm{l}$ and 2, respectively. Consequently, the difference in $\mathrm{CI}$ in $\mathrm{dB}$ can be used on HPDI during MCE to estimate the ratio of microbubble concentrations between two sites where the applied acoustic pressure is similar-for example, an increase in CI of $3 \mathrm{~dB}$ indicates a twofold increase in the ratio of bubble concentration.

\section{New quantitative method with HPDI during MCE}

The insonified ultrasound field generated during MCE is not homogeneous, ${ }^{13}$ which is principally due to attenuation by the presence of microbubbles in the path of the ultrasound beam. In the present study, $\mathrm{CI}_{\text {myo }}$ was lower in myocardial regions located deeper in the ultrasound field in the normal control subjects. This was thought to be caused by the inhomogeneity of the insonified field, in which the acoustic pressure was lower in the far field than in the near field. Therefore, it is impossible to estimate the relative microbubble concentration within the myocardium by simply comparing $\mathrm{CI}_{\text {myo }}$ between segments. We devised a new quantitative method to resolve this problem by calibrating $\mathrm{CI}_{\text {myo }}$ by using $\mathrm{CI}_{\text {blood }}$ from the adjacent blood pool in the left ventricle. As the applied acoustic pressure would be almost equal between a myocardial segment and the adjacent blood pool, the myocardium to blood ratio of microbubble concentrations can be estimated by the CI measurements at these two sites. Because the acoustic pressure is higher in the centre and less towards the edges of the image, our quantitative method may not completely compensate for these lateral variations. Although the difference in the pressure between the two close ROIs may be small and the lateral variations are expected to be substantially compensated, this issue will be investigated further in future studies.

The results of the present clinical study indicated that $\mathrm{CI}_{\text {myo }}$ on HPDI is quite variable between myocardial segments and that there is considerable interindividual variability of $\mathrm{CI}_{\text {myo }}$ from each myocardial segment. On the other hand,
RelCI varied to a much smaller degree both between myocardial segments and between individual subjects. Thus, the new quantitative method described here can overcome the changes in myocardial opacification with changes in the applied acoustic pressure. In patients with chronic coronary artery disease, RelCI of the infarcted myocardium was shown to be reduced more notably than $\mathrm{CI}_{\text {myo }}$ alone. Our findings suggest that RelCI represents the ratio of microbubble concentrations between the myocardium and intracavity blood pool independently of the applied acoustic pressure.

Theoretically, by using the new index RelCI calculated by subtracting $\mathrm{CI}_{\text {blood }}$ from $\mathrm{CI}_{\text {myo }}$ in $\mathrm{dB}$, the myocardium to blood ratio of microbubble concentrations can be estimated as $10^{\text {RelCI } / 10}$. If the microbubble concentration in the blood present in the left ventricular myocardium is equivalent to that in the intracavity blood pool, myocardial blood volume fraction can be derived as $10^{\text {RelCI/10 }} \times 100(\%)$ because the blood volume fraction of the intracavity blood is $100 \%$. In the present study, the myocardial blood volume fraction at end systole was estimated to be $3.1 \%$ from the mean RelCI in the normal control subjects. This value is not much different from the cast myocardial blood volume fraction of $4.5 \%$ reported in the pig heart, ${ }^{40}$ although morphometric data regarding myocardial blood volume in the human heart are not available. Thus, RelCI may correctly reflect myocardial blood volume fraction.

\section{Study limitations}

The in vitro and clinical experiments used only a single contrast agent and a single ultrasound apparatus. However, as the properties of each of these are relatively generic, it should be possible to extrapolate the results to other agents or instruments. Although our new quantitative method requires measurement of signals from the myocardium and from the intracavity blood pool, the dynamic range of HPDI is relatively low. Therefore, it was necessary to adjust the Doppler gain and to record two images at different gains to allow analysis of all myocardial segments. Thus, the development of newer imaging modalities with a higher dynamic range is required.

\section{Authors' affiliations}

S Yamada, K Komuro, H Onozuka, K Goto, S Fujii, A Kitabatake, Department of Cardiovascular Medicine, Graduate School of Medicine, Hokkaido University, Sapporo, Japan

T Mikami, Department of Health Sciences, School of Medicine, Hokkaido University

N Kudo, K Yamamoto, Laboratory of Biomedical Instrumentation and Measurements, Graduate School of Information Science and Technology, Hokkaido University

There are no conflicts of interest.

\section{REFERENCES}

1 Kaul S. Myocardial contrast echocardiography: 15 years of research and development. Circulation 1997;96:3745-60.

2 Wei K, Jayaweera AR, Firoozan S, et al. Quantification of myocardial blood flow with ultrasound-induced destruction of microbubbles administered as a constant venous infusion. Circulation 1998;97:473-83.

3 Skyba DM, Jayaweera AR, Goodman NC, et al. Quantification of myocardial perfusion with myocardial contrast echocardiography during left atrial injection of contrast: implications for venous injection. Circulation 1994;90:1513-21.

4 Kaul S, Jayaweera AR. Coronary and myocardial blood volumes: noninvasive tools to assess the coronary microcirculation? Circulation 1997:96:719-24.

5 Lindner JR, Villanueva FS, Dent JM, et al. Assessment of resting perfusion with myocardial contrast echocardiography: theoretical and practical considerations. Am Heart J 2000;139:231-40.

6 Andrassy P, Zielinska M, Busch R, et al. Myocardial blood volume and the amount of viable myocardium early after mechanical reperfusion of acute myocardial infarction: prospective study using venous contrast echocardiography. Heart 2002;87:350-5. 
7 Shimoni S, Frangogiannis NG, Aggeli CJ, et al. Identification of hibernating myocardium with quantitative intravenous myocardial contrast echocardiography: comparison with dobutamine echocardiography and thallium-201 scintigraphy. Circulation 2003;107:538-44.

8 Shimoni S, Frangogiannis NG, Aggeli CJ, et al. Microvascular structural correlates of myocardial contrast echocardiography in patients with coronary artery disease and left ventricular dysfunction: implications for the assessment of myocardial hibernation. Circulation 2002;106:950-6.

9 Masugata H, Cotter B, Peters B, et al. Assessment of coronary stenosis severity and transmural perfusion gradient by myocardial contrast echocardiography: comparison of gray-scale B-mode with power Doppler imaging. Circulation 2000; 102:1 427-33.

10 Becher H, Tiemann K, Schlief R, et al. Harmonic power Doppler contrast echocardiography: preliminary clinical results. Echocardiography 1997: 14:637-42.

11 Broillet A, Puginier J, Ventrone R, et al. Assessment of myocardial perfusion by intermittent harmonic power Doppler using SonoVue, a new ultrasound contrast agent. Invest Radiol 1998;33:209-15.

12 Heinle SK, Noblin J, Goree-Best P, et al. Assessment of myocardial perfusion by harmonic power Doppler imaging at rest and during adenosine stress: comparison with ${ }^{99 \mathrm{~m} T c-s e s t a m i b i}$ SPECT imaging. Circulation 2000; 102:55-60.
13 Senior R, Kaul S, Soman P, et al. Power Doppler harmonic imaging: a feasibility study of a new technique for the assessment of myocardial perfusion. Am Heart J 2000;139:245-51.

14 Muro T, Hozumi T, Watanabe $\mathrm{H}$, et al. Assessment of myocardial perfusion abnormalities by intravenous myocardial contrast echocardiography with harmonic power Doppler imaging: comparison with positron emission tomography. Heart 2003;89:145-9.

15 Villanueva FS, Gertz EW, Csikari M, et al. Detection of coronary artery stenosis with power Doppler imaging. Circulation 2001;103:2624-30.

16 Shi WT, Forsberg F. Ultrasonic characterization of the nonlinear properties of contrast microbubbles. Ultrasound Med Biol 2000;26:93-104.

17 Chin CT, Burns PN. Predicting the acoustic response of a microbubble population for contrast imaging in medical ultrasound. Ultrasound Med Biol 2000;26:1293-300.

18 Wei K, Skyba DM, Firschke C, et al. Interactions between microbubbles and ultrasound: in vitro and in vivo observations. J Am Coll Cardiol 1997;29:1081-8.

19 Schwarz KQ, Bezante GP, Chen X, et al. Quantitative echo contrast concentration measurement by Doppler sonography. Ultrasound Med Biol 1993; 19:289-97.

20 Kassab GS, Lin DH, Fung YC. Morphometry of pig coronary venous system. Am J Physiol 1994;267:H2100-13

\section{IMAGES IN CARDIOLOGY}

\section{A rare cause of ST segment elevation}

A 28 year old male patient was admitted to the emergency room after a car accident with high velocity impact. Even though the patient did not experience any chest pain, an ECG was obtained and biochemical cardiac markers were determined to exclude cardiac contusion, associated with rapid deceleration trauma. The
ECG (panel A) revealed an atrial rhythm with a PR interval of $10 \mathrm{~ms} ; 1 \mathrm{~mm}$ ST segment elevation was present in leads II, III, and aVF. Biochemical markers were within normal limits. Because the ECG abnormalities could be caused by transmural ischaemia, echocardiography was performed which did not show any wall motion abnormalities. During

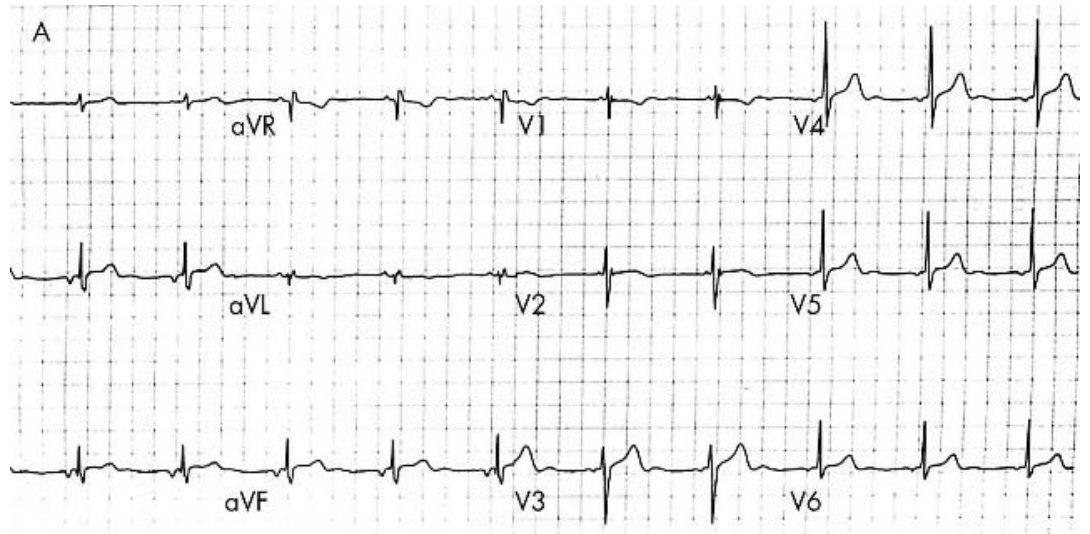

doi: 10.1136/hrt.2004.036061

observation the atrial rhythm converted spontaneously to sinus rhythm (panel B, leads VI, II, and III are shown), and immediate resolution of the ST segment elevation occurred (panel C).

The immediate resolution indicated a close correlation between the ST segment elevation and the ectopic atrial rhythm. In normal conditions, atrial repolarisation (Ta) is represented by a slow wave with a direction opposite to that of the $\mathrm{P}$ wave. Generally $\mathrm{Ta}$ is not visible in the ECG, as it is a small electrical event and is often incorporated in the QRS complex. Ectopic atrial activation inferior in the atrium results in a negative $\mathrm{P}$ wave, and consequently induces a positive Ta wave. The latter in combination with a short PR interval can modify the ST segment. Although it is a rare phenomenon, it can lead to elevation of the segment.

P Knaapen $R$ B van Loon F C Visser p.knaapen@vumc.nl
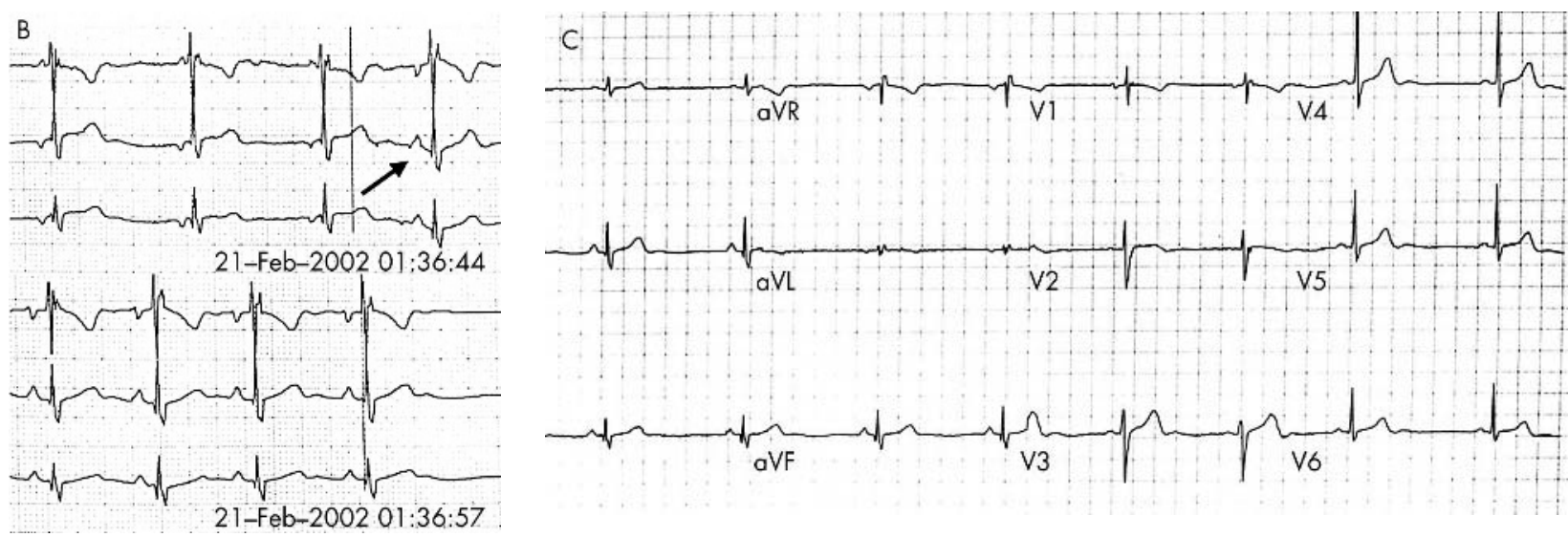